Research Paper

\title{
Distribution and Clinical Utility of the 21 -gene Recurrence Score in Pure Mucinous Breast Cancer Patients: a case-control study
}

\author{
Wei Wang1, Xiaosong Chen ${ }^{1 凶}$, Lin Lin², Xiaochun Fei ${ }^{3}$, David H. Garfield ${ }^{4}$, Jin Hong ${ }^{1}$, Weiqi Gao ${ }^{1}$, Siji
} $\mathrm{Zhu}^{1}$, Jiayi Wu${ }^{1}$, Ou Huang ${ }^{1}$, Jianrong He ${ }^{1}$, Yafen Li1 ${ }^{1}$, Li Zhu ${ }^{1}$, Weiguo Chen ${ }^{1}$, Kunwei Shen ${ }^{1}$

1. Comprehensive Breast Health Center, Ruijin Hospital, Shanghai Jiaotong University School of Medicine, 197 Ruijin Second Road, Shanghai 200025, P.R. China

2. Department of clinical laboratory, Ruijin Hospital, Shanghai Jiaotong University School of Medicine, Shanghai 200025, China

3. Pathology Department, Ruijin Hospital, Shanghai Jiaotong University School of Medicine, 197 Ruijin Second Road, Shanghai 200025, P.R. China

4. University of Colorado Comprehensive Cancer Center, Aurora, CO 80045, USA

$\square$ Corresponding authors: Dr. Xiaosong Chen, Comprehensive Breast Health Center, Ruijin Hospital, Shanghai Jiao Tong University School of Medicine, 197 Ruijin Second Road, Shanghai 200025, P.R. China. E-mail: chenxiaosong0156@hotmail.com. Tel: 8621-6437 0045-602215 and Prof. Kunwei Shen, Comprehensive Breast Health Center, Ruijin Hospital, Shanghai Jiao Tong University School of Medicine, 197 Ruijin Second Road, Shanghai 200025, P.R. China. E-mail: kwshen@medmail.com.cn. Tel: 8621-6437 0045-602208

(c) Ivyspring International Publisher. This is an open access article distributed under the terms of the Creative Commons Attribution (CC BY-NC) license (https://creativecommons.org/licenses/by-nc/4.0/). See http://ivyspring.com/terms for full terms and conditions.

Received: 2018.05.16; Accepted: 2018.07.06; Published: 2018.08.06

\begin{abstract}
The 21-gene recurrence score (RS) is increasingly being used for patients with early stage, hormone receptor-positive, Her-2-negative breast cancer. However, these results are largely from populations with infiltrating ductal carcinoma (IDC). The clinical value of RS testing in mucinous carcinoma has not been well investigated. Pure mucinous breast cancer (PMBC) and paired pure IDC patients who underwent 21 -gene RS were retrospectively reviewed and matched with tumor stage and molecular subtype. Clinic-pathological factors, treatment strategies, and RS distribution were compared between the PMBC and IDC patients. A total of 35 PMBC and 70 IDC patients were included. We found that RS was lower in the PMBC as compared with the IDC group: 21.26 vs. $24.40(P=0.037)$. Regarding $R S$ categories, $P M B C$ patients had a relatively lower percentage of high RS patients than the IDC group: $8.57 \%$ vs. $22.86 \%(P=0.048)$. Multivariate analysis showed that histologic type was an independent factor predicting RS distribution: IDC patients were associated with a higher $R S$ as compared with PMBC patients (OR: 1.27, 95\% Cl: 1.03-2.13; $P=0.014$ ). Among genes in 21-gene RS testing, HER2, STMY3, STK15, and BAGI were significantly different between the PMBC and IDC groups $(P<0.05)$. Two patients $(5.71 \%)$ in the PMBC group, both with high $R S$, were recommended to receive adjuvant chemotherapy, much lower than patients with IDC $(57.14 \%, P<$ $0.001)$. In multivariate analysis, histologic type of IDC was an independent factor for chemotherapy recommendation $(\mathrm{OR}=22.00,95 \% \mathrm{Cl}: 4.89-98.97, P<0.001)$. With a medium follow-up time of 24 months, one IDC patient had ipsilateral axillary lymph nodes recurrence and one PMBC patient had contralateral breast cancer. In conclusion, PMBC patients, mostly classified with low or intermediate $R S$ category, were associated with lower RS as compared with IDC patients. PMBC and IDC had different genes expression patterns. Patients with high RS in the PMBC group might be recommended to receive adjuvant chemotherapy, which deserves further clinical evaluation.
\end{abstract}

Key words: Breast Neoplasm, Mucinous Carcinoma, Infiltrating breast carcinoma Recurrence Score, Chemotherapy

\section{Introduction}

Breast cancer is one of the most common cancers in women. In 2013, the incidence of breast cancer among Chinese women was $41.73 / 10^{5}$, which is the most frequently diagnosed cancer in females, 
especially in urban areas [1]. Recent gene expression studies have confirmed that breast cancer is not a single disease, but a group of diseases with significant heterogeneity. Traditional clinico-pathologic factors are no longer enough to evaluate individual patient's prognosis and treatment [2]. Over the past decade, several multigene assays assessing tumor genomic profiling or molecular biomarkers have been developed to more precisely predict patients' prognosis and guide systemic therapy decisions; this includes the 21-gene recurrence score (RS, Oncotype DX) assay, Mammoprint, and PAM50.

The 21-gene RS is a multigene assay tested by the reverse transcription polymerase chain reaction (RT-PCR) method, using sections of fixed, paraffin-embedded tumor tissue. It was first validated in 2004 by to quantifying the likelihood of disease recurrence in tamoxifen-treated patients with node-negative, estrogen-receptor (ER)-positive breast cancers [3]. Further validation studies also confirmed its ability to estimate the recurrence risk in ER-positive, node-positive patients [4] and to predict the benefit from chemotherapy both in node-negative [5] and node-positive patients [6]. Recently, the latest prospective trials, TAILORx and West German Study Group Plan B trials, also revealed the prognostic and predictive value of RS in patients with early-stage, ER-positive, invasive breast cancer. Patients with RS < 11 (RS $\leq 11$ in Plan B clinical trial) had an excellent prognosis without chemotherapy [7-8] and endocrine therapy was non-inferior to chemoendocrine therapy for patients with RS 11-25 in TAILORx study [9]. These studies further confirmed the clinical value of RS and making the 21-gene test fulfill the criteria for level 1 evidence as genomic assay in various guidelines.

Based on these findings, RS has been recommended by National Comprehensive Cancer Network (NCCN) in hormone receptor (HR)-positive, HER2-negative, lymph node (LN)-negative, and $\mathrm{N}_{1 \mathrm{mi}}$ patients [10]. Its utilization can lead to changes in treatment recommendation in $20 \%$ to $70 \%$ of cases and has resulted in a $13 \%$ to $34 \%$ reduction in the use of adjuvant chemotherapy [11]. However, the precise utility and cost-effectiveness of the 21-gene RS test in guiding treatment of special types of breast cancer, such as the mucinous subtype, has not been investigated. Most clinical trials in which 21-gene RS testing was validated did not provide information about the histologic subtype [3-8]. A few small studies evaluating RS performance in breast cancer did provide histologic information, but the majority of cases (77\%-83\%) were IDC, with $8.4 \%-13 \%$ being invasive lobular carcinoma, while the remainder were other special subtypes [12-13].
Mucinous breast cancer (MBC) is a special type, comprising 1-7\% of all invasive breast cancers [14-15]. Pathologically, MBC is divided into two main subtypes, pure and mixed, based on quantification of cellularity [16]. Pure MBC (PMBC) is defined as consisting of tumor tissue with non-mucinous component being less than $10 \%$. Although this division is a matter of debate and no prognostic differences have been reported [17-18], it suggests heterogeneity in PMBC. Furthermore, there are still $5 \%-10 \%$ of PMBC patients having recurrence or metastasis within 5 years after surgery despite its relative favorable prognosis [19-20]. Given the prognostic and predictive value of recent multigene assays, we must wonder if these assays have some role in the understanding and management of PMBC.

To date, there have been no retrospective reviews or prospective studies specifically focused on the role of 21-gene RS testing in the assessment and management of patients with PMBC. Thus, we undertook this case-control study to assess the distribution pattern and treatment decision value of 21-gene RS testing in patients with HR-positive, HER2-negative, LN-negative PMBC compared with IDC in our center.

\section{Materials and Methods}

\section{Study Population}

We retrospectively reviewed information on consecutive breast cancer patients undergoing the 21-gene RS between January 2014 and December 2016 at Ruijin Hospital, Shanghai Jiaotong University School of Medicine, Shanghai, China. All medical records were retrieved from Shanghai Jiaotong University Breast Cancer Database (SJTU-BCDB). Patients with HR-positive, HER2-negative and LN-negative PMBC (mucinous variant of micropapillary carcinoma were excluded) were enrolled. Molecular subtypes were defined according to the 2013 St. Gallen Expert Consensus [21]. The definition for Luminal A-like tumor was ER positive, $\mathrm{PR} \geq 20 \%$, HER2 negative and Ki67 $<14 \%$ and the definition for Luminal B-like tumors was ER positive, HER2 negative, and PR $<20 \%$ or Ki-67 $\geq 14 \%$. To conduct this case-control study, IDC patients from the same series were matched (1:2) in terms of tumor size stage $(\mathrm{T})$, node stage $(\mathrm{N})$, and molecular subtypes (Luminal A-like vs. Luminal B-like). The protocol was approved by the Ethical Committees of Shanghai Ruijin Hospital.

\section{Evaluation of ER, PR, HER2, and Ki67 index status}

The pathology and immunohistochemistry (IHC) staining methods were reported previously 
[22]. Tumors were classified histologically according to the World Health Organization Classification of Tumors [15]. Positive staining for ER/progesterone receptor (PR) was defined as nuclear staining in $\geq 1 \%$ of tumor cells. Negative HER2 status was considered as 0 to $1+$ by IHC or negative on FISH. Ki67 index was characterized as the proportion of positive nuclear staining cells among at least 1000 tumor cells in the area counted. The following antibodies were used for the IHC test: ER: clone 1D5 (rabbit monoclonal, Gene), PR: clone PR636 (mouse monoclonal, Dako), HER2: 4B5 (rabbit monoclonal, Roche), Ki67: MIB-1 (mouse monoclonal, Dako). All histologic and IHC tumor slides were evaluated by two pathologists.

\section{1-gene RS Testing}

The RS was determined by measuring the expression of 16 cancer-related genes along with 5 reference genes and using a complex mathematical algorithm to provide a numeric score of between 0 and 100. Tests were performed from formalin-fixed, paraffin-embedded tissue as previously described [3, 22]. In brief, fixed tissues were incubated for 5 to 10 hours in 10\% neutral-buffered formalin before being alcohol-dehydrated and embedded in paraffin. RNA was extracted from two $10 \mu \mathrm{m}$ unstained sections from sufficient invasive breast cancer. Total RNA content was measured, and the absence of DNA contamination was verified. Reverse transcription of the purified RNA was carried out with the Omniscript RT kit (Qiagen, Valencia, CA); RT-PCR primers and probes were reported previously being designed using Primer Express (Applied iosystems, Foster City, CA) and Primer3 programs [23] (primers and probes were shown in supplement Table S1). Gene-specific reverse transcription was performed followed by standardized quantitative RT-PCR reactions in 96 well plates using Applied Biosystems (Foster City, CA) 7500 Real-Time PCR System. PCR cycling was performed as follows: $95^{\circ} \mathrm{C}$ for 10 minutes for one cycle, $95^{\circ} \mathrm{C}$ for 20 seconds, and $60^{\circ} \mathrm{C}$ for 45 seconds for 40 cycles. Expression of each gene was measured in triplicate and normalized relative to a set of 5 reference genes. $R S$, ranging from 0 to 100 , was derived from the reference-normalized expression measurements for the 16 cancer-related genes. Patients were then categorized into low-risk $(R S<18)$, intermediate-risk ( $R S$ 18-30), and high-risk $(R S>30)$ groups.

\section{Adjuvant treatment}

Adjuvant treatment decision for every breast cancer patient was made by a multidisciplinary team comprised of breast surgeons, medical oncologists, pathologists, radiation oncologists, and specialized breast nurses. For patients being recommended for chemotherapy, regimens included EC (epirubicin, 90 $\mathrm{mg} / \mathrm{m}^{2} \mathrm{IV}$ day 1 , and cyclophosphamide, $600 \mathrm{mg} / \mathrm{m}^{2}$ IV day 1, cycled every 21 days for 4 cycles), EC-T (epirubicin, $90 \mathrm{mg} / \mathrm{m}^{2}$ IV day 1 , and cyclophosphamide, $600 \mathrm{mg} / \mathrm{m}^{2} \mathrm{IV}$ day 1 , cycled every 21 days for 4 cycles followed by docetaxel, 80-100 $\mathrm{mg} / \mathrm{m}^{2} \mathrm{IV}$ on day 1 , cycled every 21 days for 4 cycles) and TC (docetaxel, $75 \mathrm{mg} / \mathrm{m}^{2}$ IV day 1, and cyclophosphamide, $600 \mathrm{mg} / \mathrm{m}^{2} \mathrm{IV}$ day 1 , cycled every 21 days for 4 cycles).

\section{Statistical analysis}

The Chi-square test were applied to evaluate the distribution of RS risk categories and chemotherapy recommendations among patients with different clinico-pathologic factors. Fisher's exact test was performed, when necessary. Logistic regression was used in multivariate analyses to identify risk factors associated with high $R S$ and chemotherapy recommendations. $T$ test was used to analyze distribution of gene expression levels by histologic types. All $p$ values less than 0.05 indicated statistical significance. Statistical analyses were carried out in SPSS version 17.0 (SPSS, Inc., Chicago, IL).

\section{Results}

\section{Patients and baseline clinico-pathological features}

From January 2014 to December 2016, 919 patients underwent 21-gene RS testing in our center. A total of $35 \mathrm{HR}+/ \mathrm{HER} 2-$, and LN-negative PMBC patients with 21-gene RS testing results were enrolled. For the 35 pure mucinous cancer, all patients were of Nottingham grade 1 tumors; 20 of them were hypocellular variant and 15 were hypercellular variant. Another 70 paired IDC patients were matched according to tumor stage and molecular subtype (Figure 1).

Baseline clinico-pathological features according to histologic types are shown in Table 1. Patients' characteristics were well balanced between the PMBC and IDC groups. Median age was 53 and 55 years in the PMBC and IDC groups, respectively. Breast-conserving surgery (BCS) was performed in 17 (48.57\%) patients with PMBC and 25 (35.71\%) with IDC. There were no significant differences between the PMBC and IDC groups in terms of tumor size stage, expression level of ER, PR, or Ki67. Luminal A-like patients accounted for $42.86 \%$. All patients had high expression level of ER ( $\geq 50 \%)$. In total, 31 PMBC patients were of low nuclear grade $(88.57 \%)$ and 4 were of intermediate nuclear grade (11.43\%). No PMBC patients had high nuclear grade. While most IDC patients had intermediate grade $(40 / 70,57.14 \%)$ and $17.14 \%$ had high grade $(P<0.001)$. 


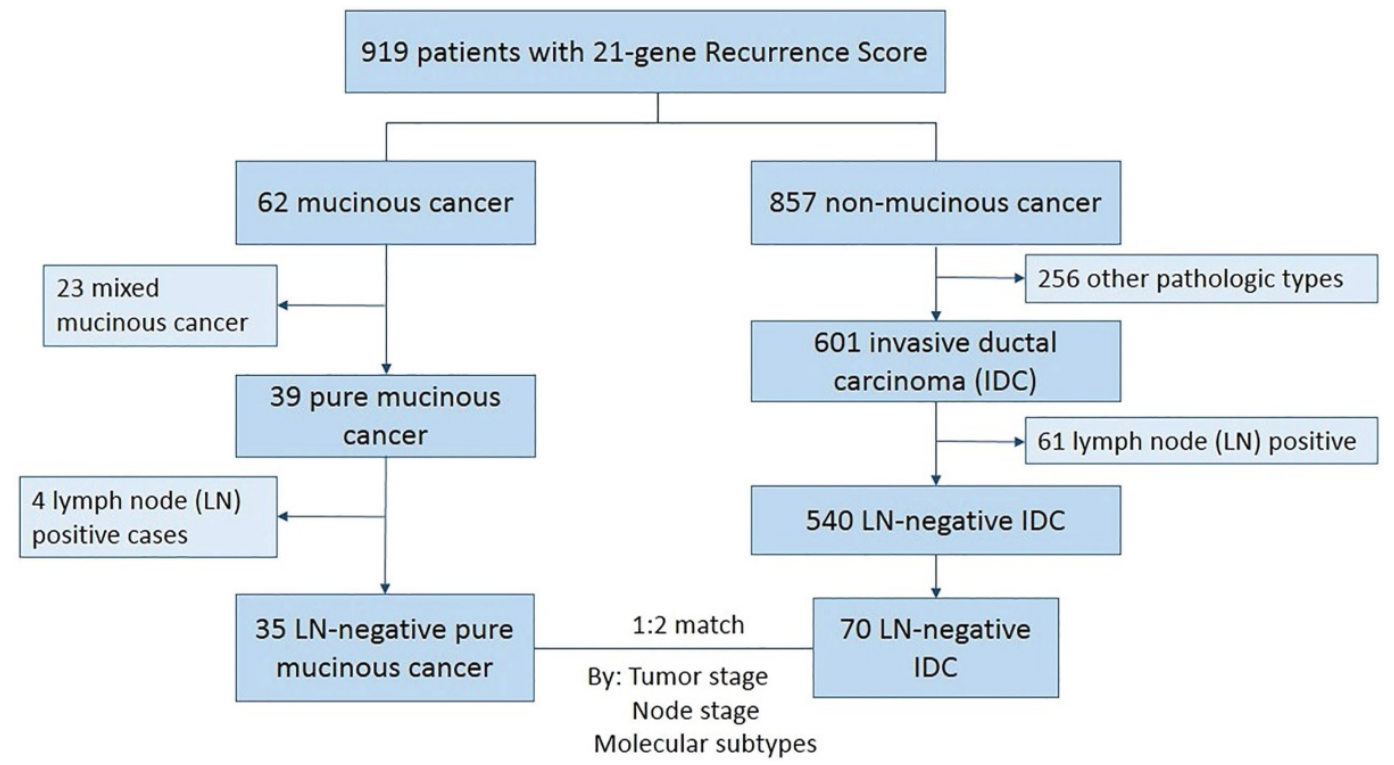

Figure 1. Patients' selection and enrollment

Table 1. Patient clinico-pathologic characteristics according to histologic types

\begin{tabular}{|c|c|c|c|}
\hline & PMBC $(n=35)$ & $\operatorname{IDC}(n=70)$ & $P$ value \\
\hline Age (median, range) & $53(27-82)$ & $55(30-81)$ & 0.992 \\
\hline Menstrual status & & & 0.165 \\
\hline Premenopausal & $19(54.29 \%)$ & $28(40.00 \%)$ & \\
\hline Menopausal & $16(45.71 \%)$ & $42(60.00 \%)$ & \\
\hline Operation type & & & 0.205 \\
\hline Mastectomy & $18(51.43 \%)$ & $45(64.29 \%)$ & \\
\hline BCS & $17(48.57 \%)$ & $25(35.71 \%)$ & \\
\hline Tumor size stage & & & 1.000 \\
\hline $\mathrm{T}_{1}$ & $18(51.43 \%)$ & $36(51.43 \%)$ & \\
\hline $\mathrm{T}_{2}$ & $17(48.57 \%)$ & $34(48.57 \%)$ & \\
\hline Nuclear grade & & & $<0.001$ \\
\hline Low & $31(88.57 \%)$ & $18(25.71 \%)$ & \\
\hline Intermediate & $4(11.43 \%)$ & $40(57.14 \%)$ & \\
\hline High & $0(0.00 \%)$ & $12(17.14 \%)$ & \\
\hline ER & & & 1.000 \\
\hline$<50 \%$ & $0(0.00 \%)$ & $0(0.00 \%)$ & \\
\hline$\geq 50 \%$ & $35(100.00 \%)$ & $70(100.00 \%)$ & \\
\hline PR & & & 1.000 \\
\hline$<20 \%$ & $14(40.00 \%)$ & $28(40.00 \%)$ & \\
\hline$\geq 20 \%$ & $21(60.00 \%)$ & $42(60.00 \%)$ & \\
\hline Ki67 & & & 1.000 \\
\hline$<14 \%$ & $24(68.57 \%)$ & $48(68.57 \%)$ & \\
\hline$\geq 14 \%$ & $11(31.43 \%)$ & $22(31.43 \%)$ & \\
\hline Molecular subtype & & & 1.000 \\
\hline Luminal A-like & $15(42.86 \%)$ & $30(42.86 \%)$ & \\
\hline Luminal B-like & $20(57.14 \%)$ & $40(57.14 \%)$ & \\
\hline RS score (mean $\pm \mathrm{SD})$ & $21.26 \pm 6.60$ & $24.40 \pm 7.43$ & 0.037 \\
\hline RS categories & & & 0.048 \\
\hline Low & $11(31.43 \%)$ & $10(14.29 \%)$ & \\
\hline Intermediate & $21(60.00 \%)$ & $44(62.86 \%)$ & \\
\hline High & $3(8.57 \%)$ & $16(22.86 \%)$ & \\
\hline
\end{tabular}

\section{Association between histologic types, clinico-pathological features, and RS}

The distribution pattern of $R S$ differed significantly between the PMBC and IDC groups (Table 1 and Figure 2). Mean value of $R S$ was $21.26 \pm$
6.60 in the PMBC group, much lower than the IDC group $(24.40 \pm 7.43, P=0.037)$. In the PMBC group, $31.43 \%$ of patients had a low RS while only 3 patients $(8.57 \%)$ had a high $R S$. For IDC patients with similar clinico-pathologic characteristics, $16(22.86 \%)$ had high $R S(P=0.048)$. In multivariable logistic regression analysis, histologic type, PR status, and nuclear grade were independent factors predicting RS distribution (Table 2). Low expression of PR $(<20 \%)$ $(\mathrm{OR}=43.11,95 \%$ CI: 3.21-579.43, $P=0.005)$ and high nuclear grade $(\mathrm{OR}=33.55,95 \% \mathrm{CI}$ : 1.05-1070.95, $P=$ 0.047 ) significantly increased the possibility of high $R S$. Whereas nuclear grade had no correlation with $R S$ category in PMBC patients $(P=0.724)$ (supplement Table S2). Patients with IDC were more likely to have high $R S$ compared with PMBC patients $(\mathrm{OR}=1.27$, 95\% CI: $1.03-2.13, P=0.014)$. ER as a continuous variable was not associated with RS category either $(P$ $=0.576$ ). The association between clinico-pathological features and RS in PMBC was also demonstrated in supplement Table S2. The results showed that younger age and Luminal B-like subtype were associated with high RS category in PMBC patients.

\section{Expression levels of genes in 21-gene RS testing between histologic types}

Among the 16-cancer associated genes in 21-gene RS testing, 4 had significantly different expression levels between the PMBC and IDC groups: HER2, STMY3, STK15, and BAG1 (Figure 3 and Supplement Table S3). PMBC had lower expression levels of HER2 ( $\triangle \mathrm{CT}-2.79$ vs. $-2.10, P=0.002)$, STMY3, ( $\triangle \mathrm{CT}-2.087$ vs. $-1.23, P<0.001)$, and STK15 $(\Delta C \mathrm{CT}-4.14$ vs. -3.62 , $P=0.025)$ compared with IDC, while expression of $B A G 1$ was much higher in the PMBC group $(\triangle C T$ 
A

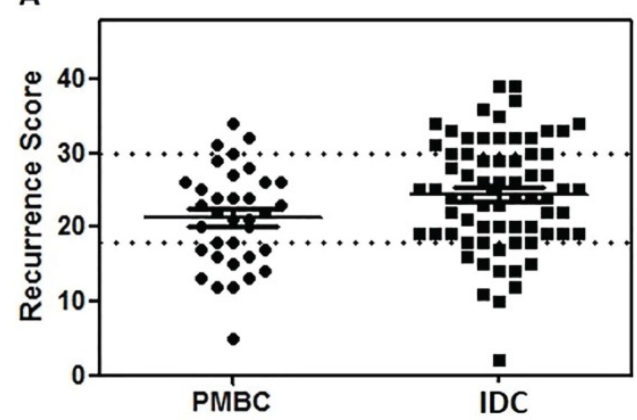

B

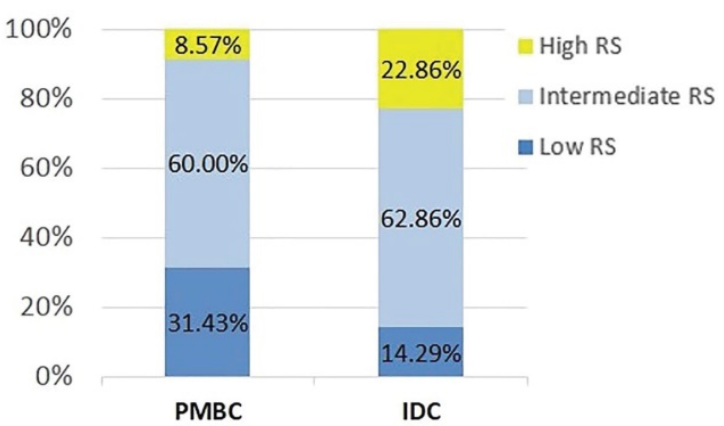

Figure 2. Distribution of Recurrence Score by histologic types. (A) Distribution of RS by histologic types; (B) Percentage of patients in different RS group. Abbreviation: $\mathrm{PMBC}=$ Pure mucinous breast cancer, IDC = Invasive ductal carcinoma, RS = Recurrence Score

-1.65 vs. $-2.25, P=0.001$ ) (Figure 4 ). For genes in the ER group, expression was similar between the PMBC and IDC groups.

Table 2. Clinical and pathological factors associated with high recurrence score.

\begin{tabular}{|c|c|c|c|}
\hline Factors & OR & $95 \% \mathrm{CI}$ & P value \\
\hline Age (years) & & & 0.619 \\
\hline$<50$ & 1 & & \\
\hline$\geq 50$ & 0.46 & $0.02-11.50$ & \\
\hline Menstrual status & & & 0.350 \\
\hline Premenopausal & 1 & & \\
\hline Menopausal & 0.21 & $0.01-5.61$ & \\
\hline Operation type & & & 0.170 \\
\hline BCS & 1 & & \\
\hline Mastectomy & 0.28 & $0.05-1.73$ & \\
\hline Histologic Type & & & 0.014 \\
\hline PMBC & 1 & & \\
\hline IDC & 1.27 & $1.03-2.13$ & \\
\hline Tumor size stage & & & 0.707 \\
\hline $\mathrm{T}_{1}$ & 1 & & \\
\hline $\mathrm{T}_{2}$ & 1.38 & $0.26-7.29$ & \\
\hline Nuclear grade & & & 0.047 \\
\hline Low & 1 & & \\
\hline Intermediate & 7.21 & $0.85-61.43$ & \\
\hline High & 33.55 & $1.05-1070.95$ & \\
\hline PR & & & 0.005 \\
\hline$\geq 20 \%$ & 1 & & \\
\hline$<20 \%$ & 43.11 & $3.21-579.43$ & \\
\hline Ki67 & & & 0.819 \\
\hline$<14 \%$ & 1 & & \\
\hline$\geq 14 \%$ & 1.28 & $0.16-10.57$ & \\
\hline Molecular subtype & & & 0.452 \\
\hline Luminal A-like & 1 & & \\
\hline Luminal B-like & 3.27 & $0.15-71.78$ & \\
\hline
\end{tabular}

\section{Comparison of chemotherapy between histologic types}

Table 3 documents the association between patients' characteristics and chemotherapy recommendation. In univariate analysis, IDC histologic type, high nuclear grade, low PR expression level, high Ki67, Luminal B-like subtype, and high RS were associated with chemotherapy usage. ER as a continuous variable didn't correlate with chemotherapy recommendation $(P=0.144)$. Two PMBC patients $(5.71 \%)$ and 40 with IDC (57.14\%) were recommended to receive adjuvant chemotherapy $(P<0.001)$. Figure 3 shows the chemotherapy usage in different $R S$ categories by histologic types. In the IDC group, 1 patient $(10 \%)$ with low $R S, 23(52.27 \%)$ with intermediate $R S$, and all patients with high $R S$ received chemotherapy. However, none of the PMBC patients with low or intermediate $R S$ received chemotherapy. Moreover, 2 PMBC patients with high RS (66.67\%) were recommended to receive adjuvant chemotherapy.

Table 3. Clinico-pathological features and chemotherapy recommendation

\begin{tabular}{|c|c|c|c|}
\hline & \multicolumn{2}{|c|}{ Adjuvant Chemotherapy } & \multirow[t]{2}{*}{$P$ value } \\
\hline & No $(n=63)$ & Yes $(n=42)$ & \\
\hline Age (years) & & & 0.869 \\
\hline$<50$ & $23(58.97 \%)$ & $16(41.03 \%)$ & \\
\hline$\geq 50$ & $40(60.61 \%)$ & $26(39.39 \%)$ & \\
\hline Operation type & & & 0.255 \\
\hline Mastectomy & $35(55.56 \%)$ & $28(44.44 \%)$ & \\
\hline BCS & $28(66.67 \%)$ & $14(33.33 \%)$ & \\
\hline Histologic Type & & & $<0.001$ \\
\hline PMBC & $33(94.29 \%)$ & $2(5.71 \%)$ & \\
\hline IDC & $30(42.86 \%)$ & $40(57.14 \%)$ & \\
\hline Tumor size stage & & & 0.067 \\
\hline $\mathrm{T}_{1}$ & $37(68.52 \%)$ & $17(31.48 \%)$ & \\
\hline $\mathrm{T}_{2}$ & $26(50.98 \%)$ & $25(49.02 \%)$ & \\
\hline Nuclear grade & & & $<0.001$ \\
\hline Low & $44(89.80 \%)$ & $5(10.20 \%)$ & \\
\hline Intermediate & $18(40.91 \%)$ & $26(59.09 \%)$ & \\
\hline High & $1(8.33 \%)$ & $11(91.67 \%)$ & \\
\hline PR & & & 0.012 \\
\hline$<20 \%$ & $19(45.24 \%)$ & $23(54.74 \%)$ & \\
\hline$\geq 20 \%$ & $44(69.84 \%)$ & $19(30.16 \%)$ & \\
\hline Ki67 & & & 0.001 \\
\hline$<14 \%$ & $51(70.83 \%)$ & $21(29.17 \%)$ & \\
\hline$\geq 14 \%$ & $12(36.36 \%)$ & $21(63.64 \%)$ & \\
\hline Molecular subtyp & & & 0.001 \\
\hline Luminal A-like & $35(77.78 \%)$ & $10(22.22 \%)$ & \\
\hline Luminal B-like & $28(46.67 \%)$ & $32(53.33 \%)$ & \\
\hline RS categories & & & $<0.001$ \\
\hline Low & $20(95.23 \%)$ & $1(4.77 \%)$ & \\
\hline Intermediate & $42(64.62 \%)$ & $23(35.38 \%)$ & \\
\hline High & $1(5.26 \%)$ & $18(94.74 \%)$ & \\
\hline
\end{tabular}


Table 4. Multivariable logistic regression analysis for factors associated with chemotherapy recommendation

\begin{tabular}{|c|c|c|c|}
\hline Factors & OR & $95 \% \mathrm{CI}$ & $P$ value \\
\hline Histologic Type & & & $<0.001$ \\
\hline PMBC & 1 & & \\
\hline IDC & 22.00 & $4.89-98.97$ & \\
\hline Tumor size stage & & & 0.020 \\
\hline $\mathrm{T}_{1}$ & 1 & & \\
\hline $\mathrm{T}_{2}$ & 5.80 & $1.32-25.42$ & \\
\hline Nuclear grade & & & 0.179 \\
\hline Low & 1 & & \\
\hline Intermediate & 3.99 & $0.61-26.03$ & \\
\hline High & 14.75 & $0.69-314.32$ & \\
\hline PR & & & 0.225 \\
\hline$\geq 20 \%$ & 1 & & \\
\hline$<20 \%$ & 3.05 & $0.50-18.51$ & \\
\hline Ki67 & & & 0.007 \\
\hline$<14 \%$ & 1 & & \\
\hline$\geq 14 \%$ & 12.25 & $2.00-74.83$ & \\
\hline Molecular subtype & & & 0.557 \\
\hline Luminal A-like & 1 & & \\
\hline Luminal B-like & 0.398 & $0.02-8.60$ & \\
\hline RS categories & & & $<0.001$ \\
\hline Low & 1 & & \\
\hline Intermediate & 10.95 & $1.38-86.95$ & \\
\hline High & 360.00 & $20.95-6186.45$ & \\
\hline
\end{tabular}

In multivariate analysis, histologic type of IDC remained an independent factor for chemotherapy recommendation $(\mathrm{OR}=22.00,95 \% \mathrm{CI}$ : 4.89-98.97, $P<0.001)$. Other independent factors included larger tumor size, high Ki67, and high RS (Table 4). Nuclear grade was not an independent impact factor for chemotherapy recommendation any more in multivariable logistic regression analysis $(P=0.179)$. There were only 2 PMBC patients who had high $R S$ were recommended to receive adjuvant chemotherapy: a 36-year old woman with RS 32 and a 41-year- old woman with low PR positivity (5\%) and RS 31. Both received 4 cycles of TC regimen.

\section{Patients' prognosis}

With a medium follow-up time of 24 months, two patients had relapse. One 47-year-old IDC patient had ipsilateral axillary lymph nodes recurrence at 34 months after surgery. She had $\mathrm{T}_{2}$ Luminal B-like breast cancer with intermediate $R S$ category. Another was $\mathrm{T}_{2}$ Luminal B-like PMBC patient with intermediate $R S$ category who had contralateral breast cancer at 30 months after surgery. Both of these two patients didn't receive adjuvant chemotherapy.

\section{Discussion}

The current study assessed the distribution patterns and clinical value of 21-gene RS testing in HR+/HER2-, and LN-negative PMBC patients. To the best of our knowledge, this was the first case-control study to compare the RS between the PMBC and IDC group. In our study, we found that $R S$ was lower in the PMBC group compared with the paired IDC group. Only 3 PMBC patients $(8.57 \%)$ had a high $R S$. Among genes in 21-gene RS testing, HER2, STMY3, $S T K 15$, and BAG1 had significant differences between the PMBC and IDC groups. For PMBC patients with high $R S, 66.67 \%$ (2 of 3 patients) were recommended to receive adjuvant chemotherapy.
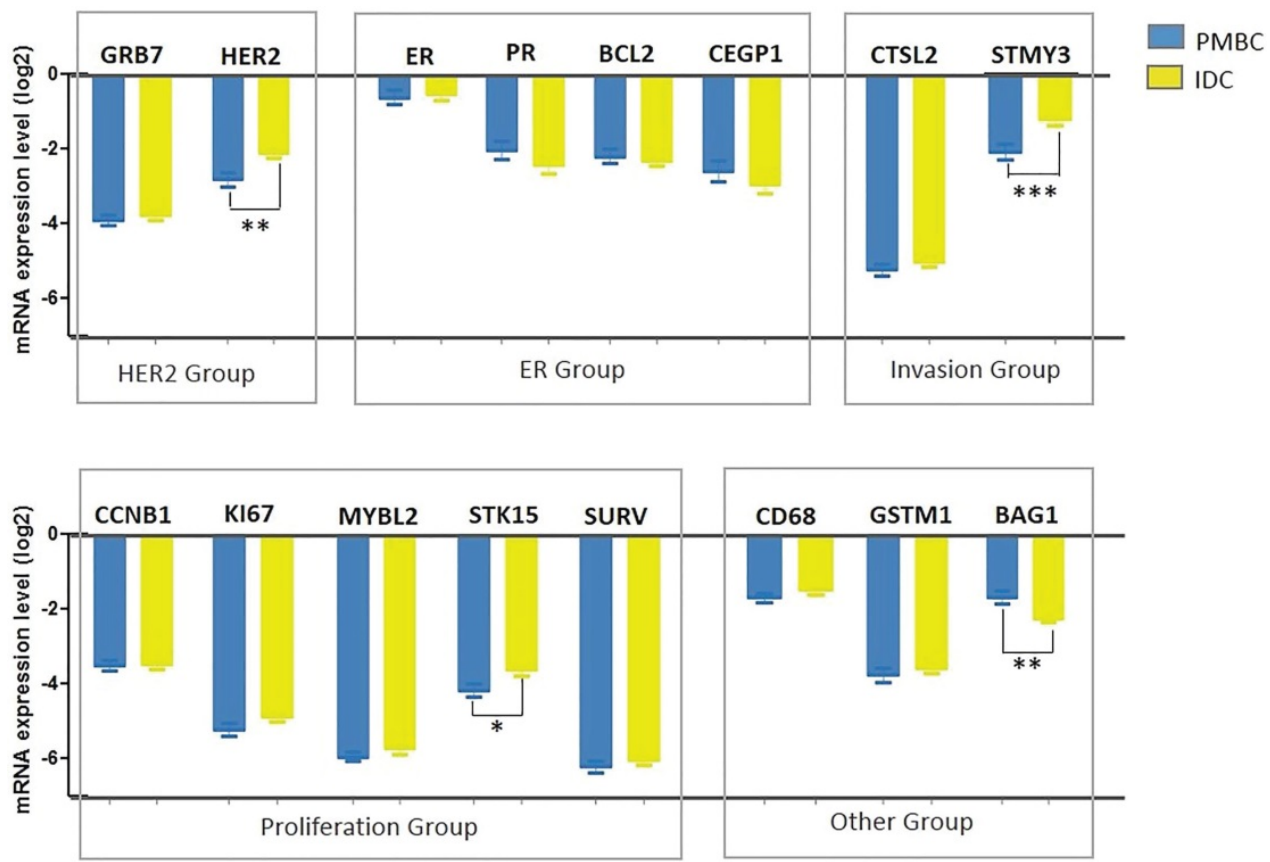

Figure 3. Distribution of genes expression levels between histologic types. $Y$ axis represents expression level of genes relative to reference genes. Abbreviation: PMBC = Pure mucinous breast cancer, IDC = Invasive ductal carcinoma 

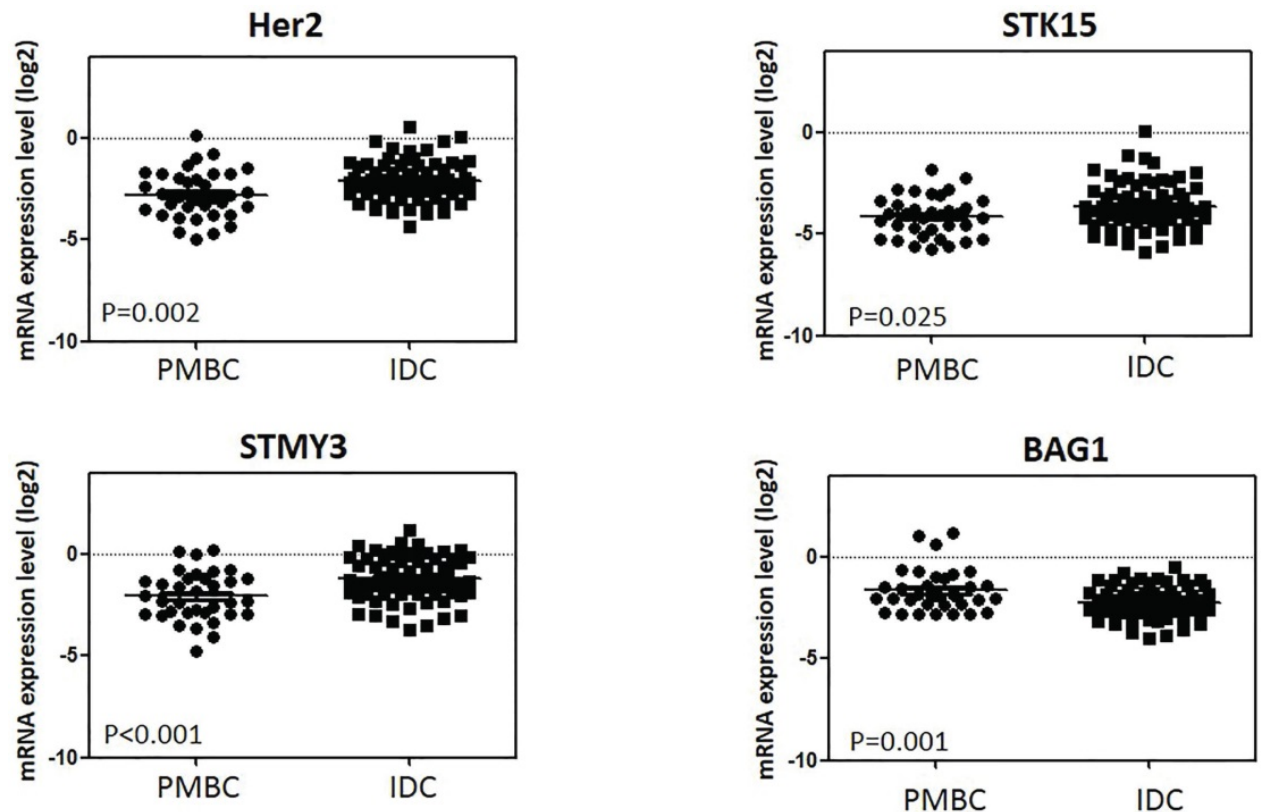

Figure 4. Genes' expression levels between two histologic types. $Y$ axis represents expression level of genes relative to reference genes. Abbreviation: $P M B C=P$ ure mucinous breast cancer, IDC = Invasive ductal carcinoma

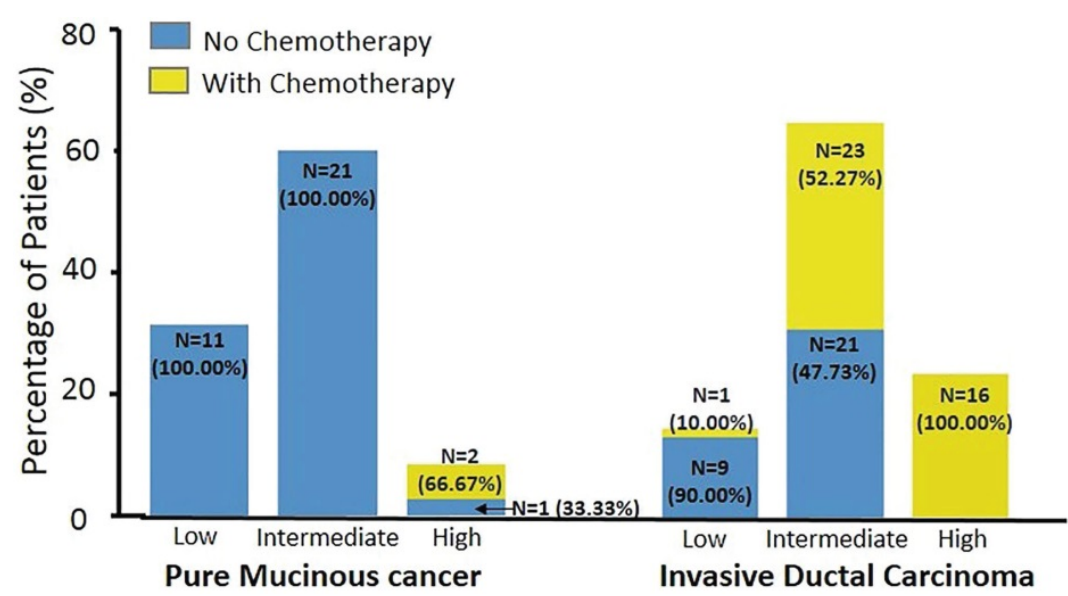

Figure 5. Distribution of chemotherapy usage between histologic types and Recurrence Score group

Previous studies have found a strong correlation between 21-gene RS and risk of recurrence and death from breast carcinoma [3-8]. The predictive value of 21-gene RS for benefit from chemotherapy has also been validated $[5,6]$. Therefore, the 21 -gene RS testing has been recommended by NCCN guidelines [10]. However, details of the histologic types tested in the early validation studies of 21-gene RS testing have not been reported. Consequently, there is a dearth of data on whether the results of these studies can be applied equally across the full range of histologic types. Two studies have investigated the correlation between 21-gene RS and histologic subtypes and found a different distribution pattern among different histologic types [24-25]. However, these studies either had very few patients with mucinous cancer (4 PMBC out of 184 enrolled cases) or did not distinguish PMBC from mixed mucinous breast cancer.
Mucinous carcinomas have been traditionally considered to be a subtype associated with a better prognosis. Recently, one group investigated the distribution of 21-gene recurrence scores in special histologic subtypes with favorable prognosis in which 33 continuous PMBC were included [26]. In this study, no PMBC patients had high RS and most $(26 / 33,78.8 \%)$ had a low RS. While in our study, the majority of PMBC $(60 \%)$ had intermediate RS category, with a low proportion had high RS (8.57\%). Since the PMBC cases in our study was not continuous and clinician tended to recommend 21-gene RS for relatively clinical high-risk patients, our study may include more high-risk cases than theirs. For example, patients in our study had younger age at diagnosis (age younger than 50 years: $45.7 \%$ vs. $36.4 \%$ ), larger tumor size (median tumor size: $2.0 \mathrm{~cm}$ vs. $1.2 \mathrm{~cm}$ ), and lower PR expression level 
(median percentage of PR by IHC: $40 \%$ vs. 90\%). Thus, this potential selection bias might contribute to the inconsistency between our results. The only other study reporting the $R S$ of mucinous cancers found that three of four had an intermediate $R S$ [24]. So, PMBC patients were more likely to have low or intermediate $R S$ and this was in accordance with its good biological behavior and prognosis.

Some traditional clinico-pathologic characteristics have been reported to be prognostic biomarkers for PMBC. In a large retrospective study which enrolled 309 PMBC patients, node positivity ( $R R=$ 6.04, 95\% CI: 1.12-13.85, $P=0.022$ ) and higher TNM stage $(\mathrm{RR}=9.07,95 \% \mathrm{CI}: 1.22-23.51, P=0.007)$ were the only independent factors for relapse-free survival in multivariate analysis [19]. Another group reported that a high Ki67 (defined as $>20 \%$ ) was related with worse 5-year disease-free survival $(\mathrm{HR}=58.72,95 \%$ CI: 1.89-1825.77, $P=0.020$ ) in PMBC [20]. In our study, we found that $8.57 \%$ of PMBC patients (3 of 35 patients) had high RS in 21-gene testing. And younger age and Luminal-B like subtype were associated with higher percentage of high $R S$ in PMBC. So, 21-gene RS may be useful in some selected PMBC patients, such as Luminal-B like, or relative young patients. However, the prognosis and chemotherapy benefit prediction role of 21-gene RS in PMBC patients was still uncertain. Besides, the cost-effectiveness study was not specifically done in PMBC to guide chemotherapy selection. Thus, it may be suggested that 21-gene RS was not necessary for every PMBC patients and further clinical study with longer follow up data for PMBC patients was needed.

Sixteen cancer-associated genes have been tested in the 21-gene RS panel. Four were differently expressed in PMBC as compared with IDC. The lower expression level of HER2 and other proliferation and invasion-related genes in PMBC might contribute to its less aggressive biological behavior. Further studies are needed to verify the influence of gene expression patterns on mucinous cancer.

Chemotherapy is not routinely recommended in $\mathrm{HR}+, \mathrm{T}_{1-3}$, and $\mathrm{LN}$-negative PMBC patients according to NCCN guidelines [10]. However, data from a large retrospective study from China showed that mucinous breast cancer patients who received chemotherapy did have better prognosis than those who didn't receive chemotherapy [19]. In our study, 3 PMBC patients had a high $R S, 2$ of whom $(66.67 \%)$ were recommended to receive chemotherapy. Thus, $R S$ may influence the clinicians' recommendation on chemotherapy, especially for PMBC patients with relatively aggressive biological behaviors (such as PR negativity or high Ki67), but further clinical evaluation is needed.
Our study has several limitations. First, it was a single-center, retrospective study with limited PMBC cases due to its relatively low incidence. Second, more than $90 \%$ PMBC patients had a low or intermediate $R S$ causing a low absolute rate of high $R S$ patients. Finally, the follow-up time was relative short. These follow-up visits are ongoing and a longer follow-up will be of benefit to draw some conclusions regarding the prognostic significance of $R S$ and its value in PMBC patients.

In conclusion, our study showed the distribution patterns of $R S$ and clinical value in HR+/HER2-, LN-negative PMBC patients. PMBC patients also had a lower $R S$ score and a less percentage of high $R S$ patients than did IDC patients. Among genes in 21-gene RS testing, HER2, STMY3, STK15, and BAG1 had a significant difference between the PMBC and IDC groups, perhaps causing low $R S$ distribution in the PMBC group. PMBC patients with high $R S$ might be recommended to receive adjuvant chemotherapy, but this warrants further clinical evaluation.

\section{Supplementary Material}

Supplementary tables.

http://www.jcancer.org/v09p3216s1.pdf

\section{Acknowledgements}

We thank Yan Fang and Yidong Du for their efforts in data entry for this study. This work was funded by National Natural Science Foundation of China (Grant Number: 81472462 and 81772797), Technology Innovation Act Plan of Shanghai Municipal Science and Technology Commission (Grant Number: 14411950200, 14411950201) and Joint Research Project of the Emerging Cutting-edge Technology of Shanghai Shen-kang Hospital Development Center (Grant Number: SHDC12014103).

\section{Competing Interests}

The authors have declared that no competing interest exists.

\section{References}

[1] Chen W, Zheng R, Zhang S, et al. Cancer incidence and mortality in China in 2013: an analysis based on urbanization level. Chin J Cancer Res. 2017; 29(1):1-10.

[2] Kwa M, Makris A, Esteva FJ. Clinical utility of gene-expression signatures in early stage breast cancer. Nat Rev Clin Oncol. 2017; 14(10):595-610.

[3] Paik S, Shak S, Tang G, et al. A multigene assay to predict recurrence of tamoxifen-treated, node-negative breast cancer. N Engl J Med. 2004; 351(27):2817-26.

[4] Dowsett M, Cuzick J, Wale C, et al. Prediction of Risk of Distant Recurrence Using the 21-Gene Recurrence Score in Node-Negative and Node-Positive Postmenopausal Breast Cancer Patients Treated with Anastrozole or Tamoxifen: A TransATAC Study. J Clin Oncol. 2010; 28(11):1829-34.

[5] Paik S, Tang G, Shak S, et al. Gene Expression and Benefit of Chemotherapy in Women with Node-Negative, Estrogen Receptor-Positive Breast Cancer. J Clin Oncol. 2006; 24(23):3726-34

[6] Albain KS, Barlow WE, Shak S, et al. Prognostic and predictive value of the 21-gene recurrence score assay in postmenopausal women with node-positive, 
oestrogen-receptor-positive breast cancer on chemotherapy: a retrospective analysis of a randomised trial. Lancet Oncol. 2010; 11(1):55-65.

[7] Sparano JA, Gray RJ, Makower DF, et al. Prospective Validation of a 21-Gene Expression Assay in Breast Cancer. N Engl J Med. 2015; 373:2005-14.

[8] Gluz O, Nitz UA, Christgen M, et al. West German Study Group Phase III PlanB Trial: First Prospective Outcome Data for the 21-Gene Recurrence Score Assay and Concordance of Prognostic Markers by Central and Local Pathology Assessment. J Clin Oncol. 2016; 34:2341-9.

[9] Sparano JA, Gray RJ, Makower DF, et al. Adjuvant Chemotherapy Guided by a 21-Gene Expression Assay in Breast Cancer. N Engl J Med. 2018. doi: 10.1056/NEJMoa1804710. [Epub ahead of print]

[10] [Internet] Network NCC. NCCN Clinical Practice Guidlines in Oncology: Breast Cancer. Version 2.2017. Available at: http://www.nccn. org/professionals/physician_gls/pdf/breast_blocks.pdf. (Accessed April 6, 2017).

[11] Hornberger J, Alvarado MD, Rebecca C, et al. Clinical validity/utility, change in practice patterns, and economic implications of risk stratifiers to predict outcomes for early-stage breast cancer: a systematic review. J Natl Cancer Inst. 2012; 104:1068-79.

[12] Joh JE, Esposito NN, Kiluk JV, et al. The effect of Oncotype DX recurrence score on treatment recommendations for patients with estrogen receptor-positive early stage breast cancer and correlation with estimation of recurrence risk by breast cancer specialists. Oncologist. 2011; 16:1520-6.

[13] Kelly CM, Krishnamurthy S, Bianchini G, et al. Utility of oncotype DX risk estimates in clinically intermediate risk hormone receptor-positive, HER2-normal, grade II, lymph node-negative breast cancers. Cancer. 2010; 116:5161-7.

[14] Anderson WF, Chu KC, Chang S, et al. Comparison of age-specific incidence rate patterns for different histopathologic types of breast carcinoma. Cancer Epidemiol Biomarkers Prev. 2004; 13: 1128-1135.

[15] Di Saverio S, Gutierrez J, Avisar E. A retrospective review with long term follow up of 11,400 cases of pure mucinous breast carcinoma. Breast Cancer Res Treat. 2008; 111: 541-547.

[16] Lakhani SR, Ellis IO, Schnitt SJ, Tan PH, van de Vijver MJ. WHO classification of tumours of the breast. In: World Health Organization classification of tumours. 4th ed. Lyon: International Agency for Research on Cancer; 2012:60-61.

[17] Ranade A, Batra R, Sandhu G, et al. Clinicopathological evaluation of 100 cases of mucinous carcinoma of breast with emphasis on axillary staging and special reference to a micropapillary pattern. J Clin Pathol. 2010; 63:1043-7.

[18] Kashiwagi S, Onoda N, Asano $Y$, et al. Clinical significance of the sub-classification of 71 cases mucinous breast carcinoma. Springerplus. 2013; 2:481-7.

[19] Cao AY, He M, Liu ZB, et al. Outcome of pure mucinous breast carcinoma compared to infiltrating ductal carcinoma: a population-based study from China. Ann Surg Oncol. 2012; 19(9):3019-27.

[20] Pan B, Yao R, Shi J, et al. Prognosis of subtypes of the mucinous breast carcinoma in Chinese women: a population-based study of 32-year experience (1983-2014). Oncotarget. 2016; 7(25):38864-38875.

[21] Goldhirsch A, Winer EP, Coates AS, et al. Personalizing the treatment of women with early breast cancer: highlights of the St Gallen International Expert Consensus on the Primary Therapy of Early Breast Cancer 2013. Ann Oncol. 2013; 24:2206-23.

[22] Wu JY, Fang Y, Lin L, et al. Distribution patterns of 21-gene recurrence score in 980 Chinese estrogen receptor-positive, HER2-negative early breast cancer patients. Oncotarget. 2017; 8(24):38706-38716.

[23] Cronin M, Sangli C, Liu ML, et al. Analytical validation of the Oncotype DX genomic diagnostic test for recurrence prognosis and therapeutic response prediction in node-negative, estrogen receptor-positive breast cancer. Clin Chem. 2007; 53(6):1084-91.

[24] Bomeisl PE, Thompson CL, Harris LN, et al. Comparison of Oncotype DX Recurrence Score by Histologic Types of Breast Carcinoma. Arch Pathol Lab Med. 2015; 139(12):1546-9.

[25] Hanna MG, Bleiweiss IJ, Nayak A, et al. Correlation of Oncotype DX Recurrence Score with Histomorphology and Immunohistochemistry in over 500 Patients. Int J Breast Cancer. 2017; 2017:1257078.

[26] Turashvili G, Brogi E, Morrow M, et al. The 21-gene recurrence score in special histologic subtypes of breast cancer with favorable prognosis. Breast Cancer Res Treat. 2017; 165(1):65-76. 